\title{
Generation of intense continuum extreme-ultraviolet radiation by many-cycle laser fields
}

\author{
P. TZALLAS ${ }^{1 *}$, E. SKANTZAKIS², C. KALPOUZOS ${ }^{1}$, E. P. BENIS ${ }^{1}$, G. D. TSAKIRIS ${ }^{3}$ \\ AND D. CHARALAMBIDIS 1,2
}

\author{
${ }^{1}$ Foundation for Research and Technology - Hellas, Institute of Electronic Structure \& Laser, PO Box 1527, GR71110 Heraklion (Crete), Greece \\ ${ }^{2}$ Department of Physics, University of Crete, PO Box 2208, GR71003 Heraklion (Crete), Greece \\ ${ }^{3}$ Max-Planck-Institut für Quantenoptik, D-85748 Garching, Germany \\ *e-mail: ptzallas@iesl.forth.gr
}

Continuing efforts in ultrashort pulse engineering have recently led to the breakthroughs of the generation of attosecond $\left(10^{-18} \mathrm{~s}\right)$ pulse trains $\mathrm{s}^{1-7}$ and isolated pulses ${ }^{8-11}$. Although trains of multiple pulses can be generated through the interaction of many-optical-cycle pulses with gases-a process that has led to intense extreme-ultraviolet emission ${ }^{3-5}$ - the generation of isolated high-intensity pulses, which requires few-cycle driving pulses, remains a challenge. Here, we report a vital step towards the generation of such pulses, the production of broad continuum extreme-ultraviolet emission using a high-intensity, many-cycle, infrared pulsed laser, through the interferometric modulation of the ellipticity of 50 -fs-long driving pulses. The increasing availability of high-power many-cycle lasers and their potential use in the construction of intense attosecond radiation-with either gas or solid-surface targets ${ }^{12}$ - offer exciting opportunities for multiphoton extreme-ultravioletpump-extreme-ultraviolet-probe studies of laser-matter and laser-plasma interactions.

The generation of attosecond pulse trains through the synthesis of a comb of harmonics of an infrared many-cycle femtosecond laser pulse is well established ${ }^{1-7}$, and is essentially understood in the framework of the three-step model ${ }^{13,14}$. According to this model, an electron is ejected in the continuum after tunnelling through the atomic potential barrier formed by the instantaneous laser field. Subsequently, the electron accelerates away from the core until the field changes sign. Within a fraction of half the laser period, the electron may revisit the parent ion to recombine and emit a burst of continuum extreme-ultraviolet radiation. As the process is repeated twice per laser cycle, the emitted spectrum consists of a superposition of coherent continua, which in the time domain is equivalent to a train of sub-femtosecond pulses. Using high-power many-cycle laser pulses, intense attosecond pulse trains have been generated and already used for the study of nonlinear phenomena in the extreme-ultraviolet spectral region ${ }^{15-22}$. They thus afford the means for the temporal characterization of attosecond pulses on the basis of second-order autocorrelation techniques in the extreme-ultraviolet spectral region ${ }^{3-5,18,19}$, and open the road towards extreme-ultraviolet pump-extremeultraviolet probe experiments.
In the spirit of the three-step model, if the process is confined to a single revisit of the core by the driven electron, one single continuum is emitted in the form of an isolated pulse. In mathematical terms, the Fourier synthesis of a broad continuum corresponds in the time domain to a single temporal occurrence, whereas a discrete spectrum leads to a repetitive process. Thus, the emission of a single coherent continuum is an essential prerequisite for single attosecond pulse generation ${ }^{8-10}$. Indeed, the generation of isolated single attosecond pulses is based on the synthesis of a continuum extreme-ultraviolet radiation emitted, so far, by rare gases driven by few-cycle infrared laser fields. Emission ensues only during a small fraction of the highest amplitude optical cycle of the driving laser field, either by selecting the highest energy part of the spectrum $^{9}$, or through the temporal modulation of the laser field ellipticity confining the linear polarization and thus the extremeultraviolet emission at the pulse centre ${ }^{23}$. In both techniques, the stabilization of the relative carrier-envelope phase (CEP) is highly pertinent $t^{24}$. Such isolated attosecond pulses are, so far, less intense and contain notably less photons than attosecond pulse trains.

The highly desirable generation of intense single attosecond pulses remains a formidable quest. Towards this goal, two different approaches are currently considered: either by developing high-power few-cycle laser systems or, starting with existing high-peak-power many-cycle laser pulses, by isolating half a laser period, during which intense extreme-ultraviolet radiation is emitted. The second approach can be implemented through the modulation of the ellipticity of the many-cycle pulse, a technique proposed by Corkum et al. ${ }^{23}$, established as 'polarization gating'. Owing to the strong dependence of the harmonic generation on the ellipticity $(\varepsilon)$ of the driving laser field, an ellipticity-modulated laser pulse, acquiring linear polarization only at the pulse centre, provides an ultrashort time interval, during which the harmonic emission essentially takes place. In this way, a broadband single attosecond pulse can be formed. Using a different polarization-gating approach than that proposed by Corkum et al., and CEP-stabilized $5 \mathrm{fs}$ laser pulses, Sola et al. ${ }^{11}$ succeeded in controlling the electron wavepacket dynamics and thus generating tunable continuum extreme-ultraviolet radiation. Their approach uses a very compact and simple to operate arrangement but 


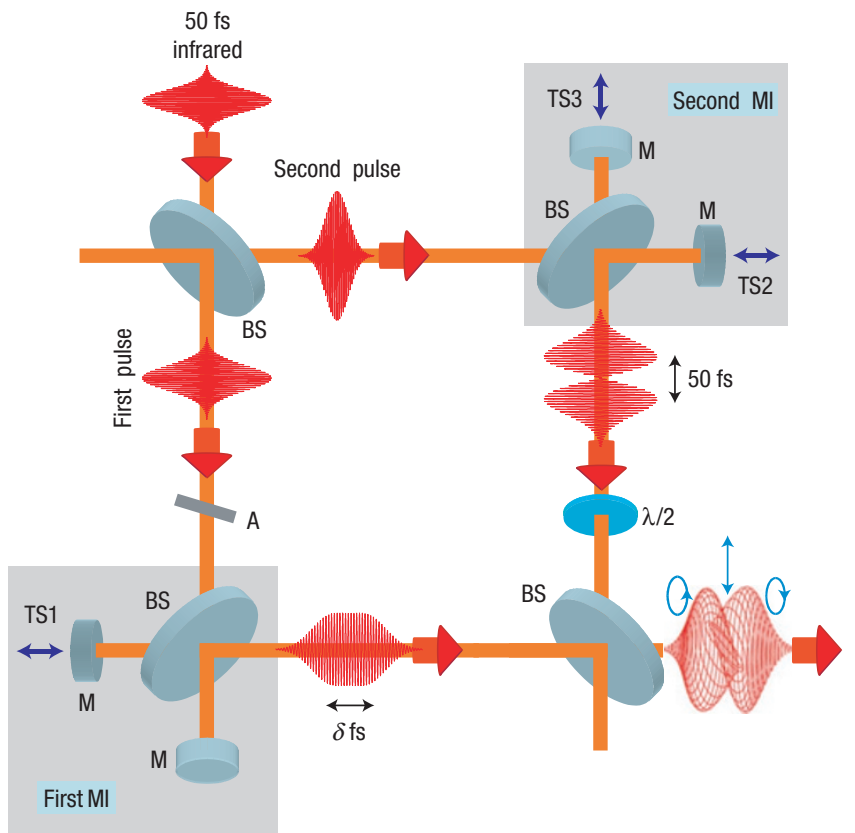

Figure 1 Dual Michelson interferometer device. BS: beam splitters. M: flat mirrors. TS1,2,3: piezoelectric translation stages. A: intensity attenuator. First and second Ml: first and second Michelson interferometers.

falls short in the efficient generation of intense continuum extreme-ultraviolet radiation by many-cycle high-power lasers ${ }^{25}$.

In our approach, which we refer to as 'interferometric polarization gating, the superposition of four many-cycle laser fields has been used to create and control the time interval during which efficient harmonic generation occurs. This interval is quantified by the temporal gate parameter $\tau_{\mathrm{g}}$, during which the ellipticity, $\varepsilon$, varies from zero to $\varepsilon_{\text {th }}$, where $\varepsilon_{\text {th }}$ is the ellipticity at which the harmonic efficiency drops to half of its maximum value, that is, its value at $\varepsilon=0$. The gate is synthesized by the four fields using a double Michelson interferometer (Fig. 1) and is tailored by independently controlling their relative phase, amplitude and polarization (see the Methods section). In this way, the gate window has been successfully confined to widths that lead to the generation of a continuum extreme-ultraviolet radiation spectrum. Introducing appropriate delays between the four fields, two of them are brought to partial overlap interfering constructively at the exit of the first Michelson interferometer, whereas the other two synthesize a destructive interference minimum at the centre of the pulse exiting the second Michelson interferometer. The linear polarizations of the two outgoing pulses are set to be perpendicular to each other and the pulse durations and intensities are appropriately adjusted. On recombination of these pulses, the temporal ellipticity modulation of the final outgoing beam can be tailored at will, thus providing a fully controllable $\tau_{\mathrm{g}}$ at optimum output power. Control parameters are (1) the ratio $I_{1} / I_{2}$ of the intensities entering the two interferometers and (2) the delay, $\delta$, introduced by the first Michelson interferometer (variable in multiples of the laser period for constructive interference). The delay in the second Michelson interferometer is kept constant at $50.7 \mathrm{fs}$.

The contour plots of Fig. 2 show $\tau_{\mathrm{g}}$ and the intensity ratio $I_{\mathrm{g}} / I_{\mathrm{in}}$, where $I_{\mathrm{g}}$ is the intensity of the driving laser field within $\tau_{\mathrm{g}}$ and $I_{\text {in }}$ is the total incoming intensity, respectively, as a function of $\delta$
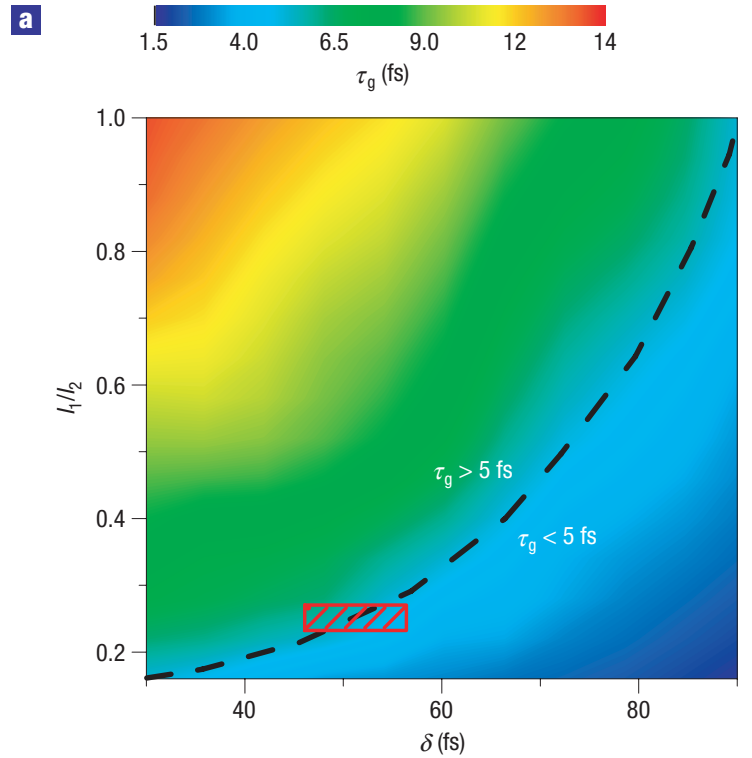

b
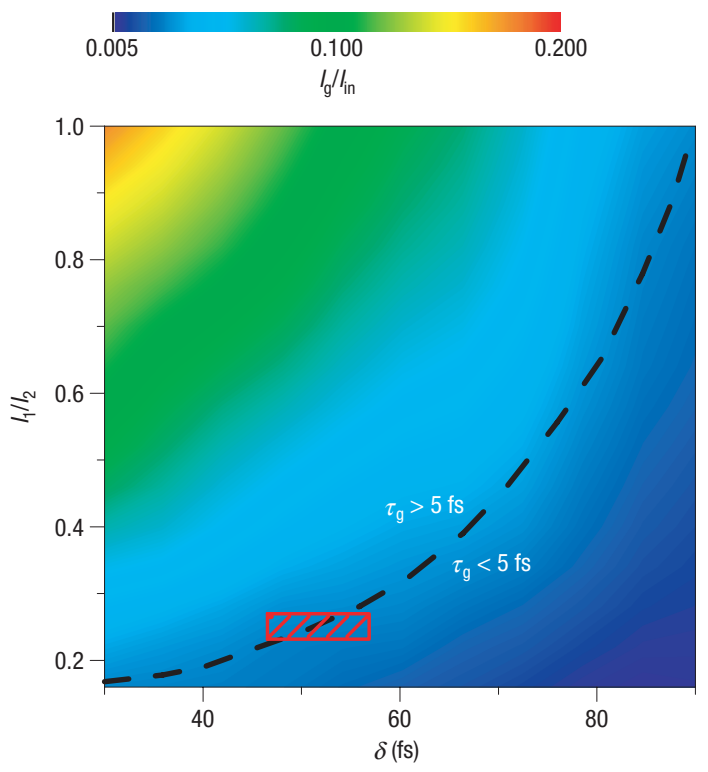

Figure 2 Polarization gating parameter definitions and gate specifications. a, Contour colour plot on a linear scale showing the dependence of the time gate width $\left(\tau_{\mathrm{g}}\right)$ on the time delay, $\delta$, between the two interfering pulses of the first Michelson interferometer, for different ratios, $l_{1} / I_{2}$, of the intensities entering the first and second interferometers. $\mathbf{b}$, Contour colour plot on a linear scale showing the intensity ratio $I_{g} / I_{\text {in }}$ (where $I_{g}$ is the intensity of the driving laser field within $\tau_{g}$ and $l_{\text {in }}$ is the total incoming intensity) as a function of $\delta$ for different intensity ratios, $I_{1} / I_{2}$. In both plots, the phase difference between the two perpendicularly polarized outgoing fields is $\Delta \varphi=\pi / 2$, the threshold ellipticity $\varepsilon_{\text {th }}$ is set to $15 \%$ and the pulse duration of each of the beams is $50 \mathrm{fs}$. The black dashed line shows the locus of the $5 \mathrm{fs}$ gate width. The red shaded area indicates the parameter range used in the experiment.

and $I_{1} / I_{2}$. The phase difference between the two perpendicularly polarized fields is $\Delta \varphi=\pi / 2$ and the threshold ellipticity $\left(\varepsilon_{\text {th }}\right)$ for the harmonic generation has been assumed to be $15 \%$ (ref. 11). The dashed black lines divide both plots into two regions, with $\tau_{\mathrm{g}}>5 \mathrm{fs}$ and $\tau_{\mathrm{g}}<5 \mathrm{fs}$, respectively. 


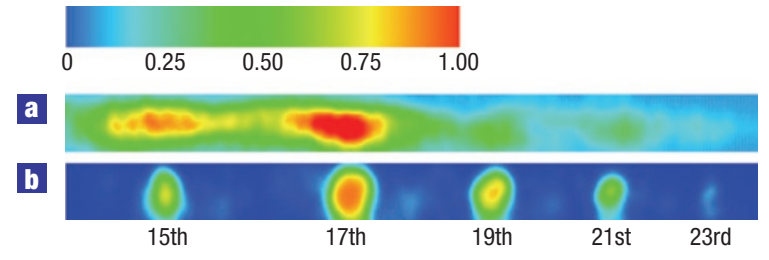

$\mathbf{G}$

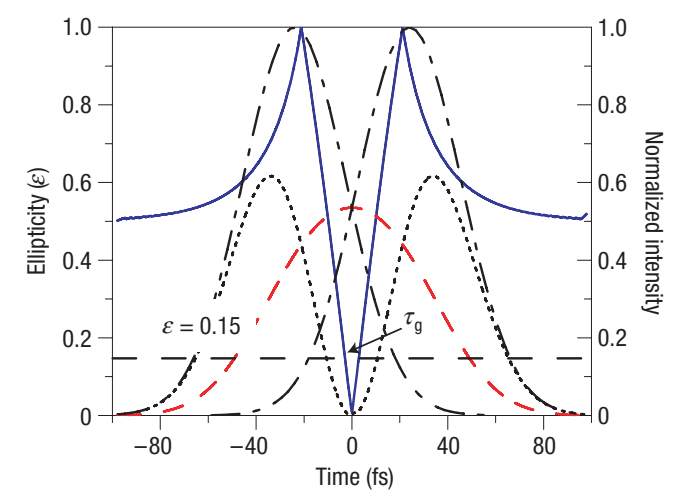

Figure 3 Generation of a continuum extreme-ultraviolet spectrum with a $5 \mathrm{fs}$ gate width. a, Iso-intensity colour plot on a linear scale showing the continuum spectrum generated by an elliptically modulated pulse possessing almost linear polarization in a time gate of $\sim 5 \mathrm{fs}$ width. $\mathbf{b}$, Iso-intensity colour plot on a linear scale showing a discrete spectrum of harmonics generated by a linearly polarized pulse. c, Solid blue line: ellipticity of the polarization-modulated pulse used for the generation of the continuum extreme-ultraviolet radiation. Black dashed-dotted line: normalized intensity of the two pulses, which partially overlap to form a pulse with a destructive interference minimum in its central part (black dotted line). Red dashed line: intensity distribution of the pulse showing the constructive interference maximum. The threshold ellipticity $\varepsilon_{\text {th }}=15 \%$ and the time gate width $\tau_{\mathrm{g}} \sim 5 \mathrm{fs}$ are shown.

The output beam is focused into an Ar gas jet, where coherent extreme-ultraviolet emission occurs. Using only one beam, a discrete spectrum with well-separated harmonics (15th to 25th) has been recorded. Using all four beams and choosing the parameters $\delta$ and $I_{1} / I_{2}$ so that $\tau_{\mathrm{g}}$ and $I_{\mathrm{g}} / I_{\mathrm{in}}$ are in the range defined by the red shaded area in Fig. 2a,b, a continuum harmonic spectrum spanning from 55 to $32 \mathrm{~nm}$ has been recorded (contour plot in Fig. 3a). A displacement of the mirror by $\lambda / 8$ in one of the arms of the first Michelson interferometer leads to a discrete harmonic spectrum consisting of the well-separated 15th to 23rd harmonics (contour graph in Fig. 3b).

Figure $3 \mathrm{c}$ shows the calculated ellipticity of the polarizationmodulated pulse and the relative intensities of the two perpendicularly polarized fields at the output of the device, for the case of the continuum extreme-ultraviolet generation (Fig. 3a). The width of the time gate is $\tau_{\mathrm{g}} \sim 5 \mathrm{fs}$ and the intensity ratios $I_{1} / I_{2}$ and $I_{\mathrm{g}} / I_{\text {in }}$ are 0.25 and 0.035 , respectively. The red and blue solid lines in the spectra of Fig. 4a show the extreme-ultraviolet signal of the contour plots of Fig. 3a and b (the central cut), respectively. Each spectrum is an average of 300 shots.

As the laser system used is not CEP stabilized, the latter result does not warrant emission of isolated pulses and thus has to be further scrutinized. The drifting of the CEP in the present experiment is reflected in the extreme-ultraviolet spectra of Fig. $4 \mathrm{~b}$. Each spectrum in this figure is the record of a single shot. The spectrum shown with the black solid line is a quasi-continuum, whereas the spectra shown with the red dashed and blue dotted
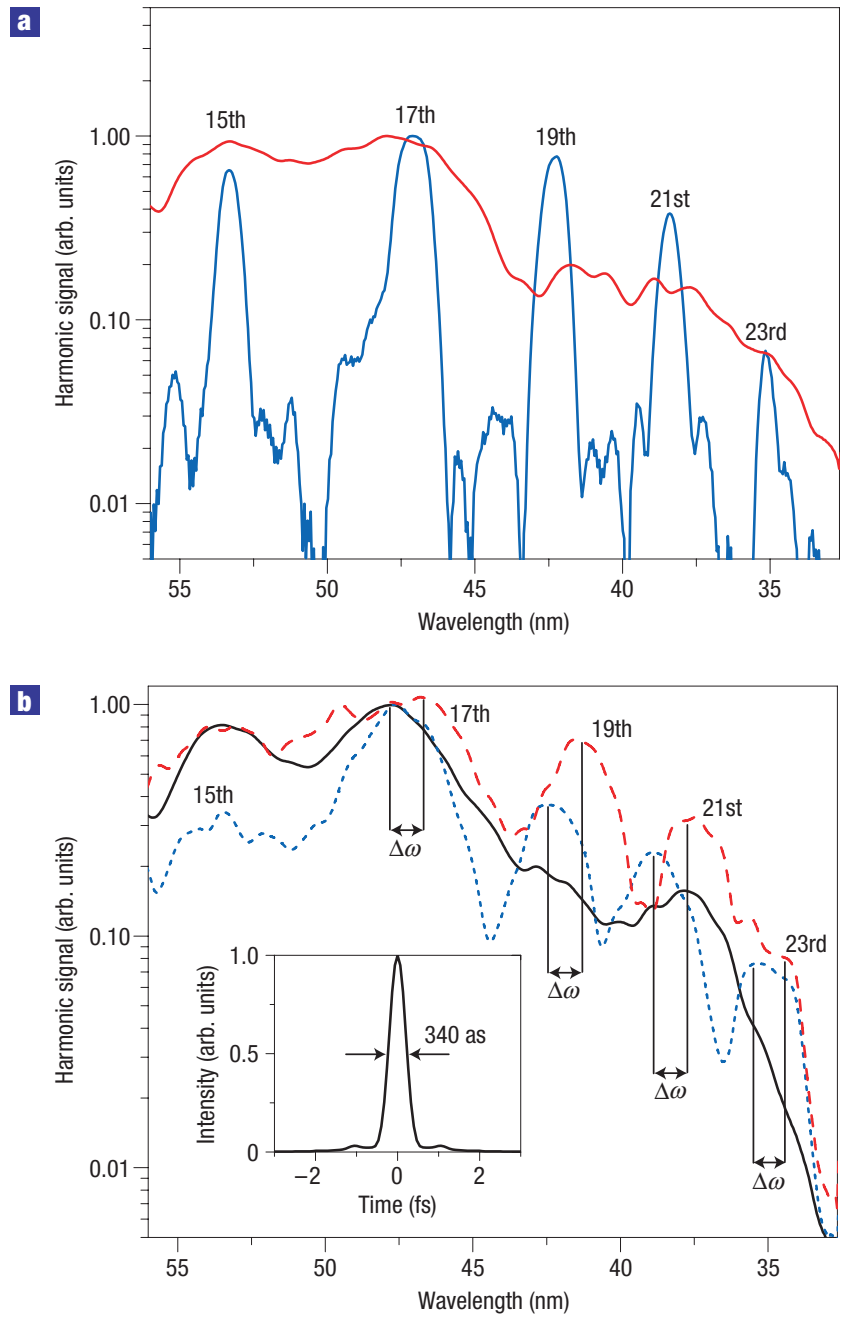

Figure 4 Dependence of the extreme-ultraviolet spectrum on the CEP of the laser pulse. a, Blue solid line: normalized harmonic spectrum generated by a linearly polarized pulse. Red solid line: normalized continuum extreme-ultraviolet spectrum generated by the central part of the elliptically modulated pulse, that is defined by the time window $\tau_{\mathrm{g}} \sim 5 \mathrm{fs}$. Each spectrum is an average of 300 shots. b, Evidence of the CEP variation, reflected in the different structure of the spectra and the frequency shift $(\Delta \omega)$ between the harmonic peaks. Solid black line: normalized extreme-ultraviolet spectrum generated by a half cycle of the driving laser field. Red dashed and blue dotted lines: normalized extreme-ultraviolet spectra, each one generated by one cycle of the driving laser field with different CEPs. The inset shows the calculated temporal profile of the Fourier-transform-limited single attosecond pulse resulting from the 55 to $32 \mathrm{~nm}$ spectral range of the spectrum, transmitted by a Si filter.

lines are of a discrete nature. Furthermore, a clear frequency shift $(\Delta \omega)$ of the harmonic peaks in the two different discrete spectra is observable. All of the above differences in the graphs of Fig. $4 \mathrm{~b}$ are indicative of a different CEP in each spectrum. The influence of the CEP drift on the spectra of the three recordings is compatible with previous findings in experiments conducted with CEP-stabilized few-cycle pulses ${ }^{24}$. The spectrum shown with a black solid line can be attributed to have been generated by a half cycle of a 'cosine'-type driving laser field, whereas the other two spectra generated by one cycle of a 'non-cosine' driving laser field with different CEPs. Using a Si filter transmitting between 55 and $32 \mathrm{~nm}$, a single attosecond 
pulse can be isolated with a minimum duration of $\sim 340$ as (inset in Fig. 4b).

It is worth noting that the full interferometric control of the parameters used here enables the creation of an ultrashort temporal gate with the highest possible energy content, which may reach several millijoules. Such gates either cannot be achieved or require strikingly longer delays when produced by previously mentioned methods ${ }^{11}$, forcing as a result the gate formation to occur at the far wings of the pulses, thus substantially reducing its energy content. Moreover, our approach is not based on refractive optical components and thus is sensitive neither to the initial laser pulse length nor to nonlinear effects occurring through propagation of high intensities. Application of polarization gating to many-cycle pulses is inevitably connected to a large percentage of energy waste. In general, for the same laser energy input and given gate width, the shorter the laser pulse the higher the energy content in the gate. The followed approach can be optimized with respect to the percentage of energy losses using shorter pulses with a duration of 10-20 fs for which CEP stabilization technology already exists. Notwithstanding this optimization there will be a reduction of the overall output power in the gate because so far the highest gate power content that can be achieved is with longer pulses only from few-10 TW laser systems. This constraint will be eased in the future when highenergy CEP pulses will be available. Nevertheless, the method is versatile and applicable to a large range of pulse durations and thus may serve different specific needs.

In summary, we have presented a novel approach for intense broadband extreme-ultraviolet continuum generation using highpeak-power many-cycle pulses. The generated bandwidth is as broad as to enable the synthesis of isolated extreme-ultraviolet pulses with pulse durations of a few hundred attoseconds. For a large number of existing high-peak-power laser installations, the present results open up exciting prospects for the generation of intense, tunable in duration and frequency, isolated attosecond extreme-ultraviolet pulses. In particular, the approach is applicable without any conceptual modification to the extreme-ultraviolet generation from laser surface plasma interactions at relativistic intensities ${ }^{12}$, aiming at the generation of isolated attosecond pulses with unprecedented extreme-ultraviolet intensities ${ }^{26}$. Although so far CEP has never been considered as an important parameter when many-cycle pulses are used, this work has revealed its importance to the generation of extreme-ultraviolet continua. This is because of the very narrow time gate formed by the transient linear polarization, which is effectively equivalent to using a few-cycle driving field. Exploitation of the approach in extreme-ultravioletpump-extreme-ultraviolet-probe applications will thus further require either stabilization of the CEP or tagging the recorded spectra with a simultaneously measured quantity (for example, the frequency spectrum) indicative of the shot-to-shot CEP. With these developments, unique extreme-ultraviolet radiation sources will become available, providing the highest ever temporal resolution at competitive intensities.

\section{METHODS}

The laser system used is a $10 \mathrm{~Hz}$ Ti:sapphire, delivering $50 \mathrm{fs}$ pulses, with an energy of up to $150 \mathrm{~mJ}$ per pulse and a carrier wavelength of $800 \mathrm{~nm}$. The laser beam is split into two parts by a beam splitter (see Fig. 1) and the resulting two pulses enter the double Michelson arrangement. One of the beams (first pulse) enters the first Michelson interferometer, introducing a multiple of the laser period time delay $(\delta)$ between the two arms, so that the output pulse possesses a constructive interference maximum at its centre. The other beam (second pulse) enters the second Michelson interferometer, where the delay is set to a fixed value of about $50 \mathrm{fs}$. Through fine-tuning of this delay, an output pulse marked by a destructive interference minimum at the centre is tailored. The $50 \mathrm{fs}$ delay ensures the highest possible sharpness of the interference minimum allowed by the temporal envelope of the original pulse. The linear polarizations of the two outgoing beams are adjusted to be perpendicular to each other by a $\lambda / 2$ zero-order waveplate. With this arrangement, the temporal ellipticity modulation of the outgoing superposition of the two pulses can be tailored at will, thus providing a fully controllable $\tau_{\mathrm{g}}$. The attenuator placed before the first Michelson interferometer, together with the variable delay of the first Michelson interferometer, serves to adjust the intensity distributions of the two outgoing pulses to result in an optimal combination of gate width and gate energy content avoiding any gate formation at the tails of the tailored pulse. This optimal combination can only be achieved through an independent manipulation of the two fields, here realized by the dual Michelson interferometer, and constitutes a significant advantage of the method. However, conditions close to the optimal ones can be achieved using the same delay but different amplitudes in each pair (one undergoing constructive and the other destructive interference) of pulses. The set-up can then be substantially simplified, increasing its throughput at the same time by a factor of 2 . This possible modification will be presented elsewhere. On the basis of the calculated dependence of $\tau_{\mathrm{g}}$ and $I_{\mathrm{g}} / I_{\text {in }}$ on $\delta$ and $I_{1} / I_{2}$ (Fig. 2a,b), the $\delta$ and $I_{1} / I_{2}$ parameter values have been chosen to result in a gate width $\tau_{\mathrm{g}} \sim 5 \mathrm{fs}$ and a ratio $I_{\mathrm{g}} / I_{\text {in }} \sim 0.035$ (red shaded area in Fig. 2a,b).

The output tailored beam is focused into a piezoelectrically pulsed Ar gas jet, where the harmonic generation is taking place. The beam focus is placed before the Ar gas jet to select the short electron trajectory ${ }^{27,28}$. The harmonic radiation is monitored by an extreme-ultraviolet toroidal grating monochromator in a grazing-incident configuration, equipped with an imaging detector, coupled to a charge-coupled device (CCD) camera capable of recording single shot measurements.

It is worth noting that the longer the initial pulse duration is the more advantageous our method becomes. For pulse durations of $\leq 30 \mathrm{fs}$, our method has advantages only for high driving intensities at which induced nonlinear effects prohibit the use of refractive materials, owing to the accumulation of high B-integral values. For pulses with a duration of $\geq 50 \mathrm{fs}$, it becomes superior to other methods, if not the only alternative. As most of the high-peak-power (10-100 TW) laser systems deliver pulses with a duration of $\geq 40 \mathrm{fs}$, the use of our method in these systems provides a unique tool for intense isolated attosecond pulse generation. In this context, a current limiting factor is the lack of CEP-stabilized high-peak-power systems. Efforts towards such CEP stabilization are in progress in a number of large laser laboratories, but the timescale necessary for its implementation cannot be precisely foreseen. Awaiting this accomplishment, extreme-ultraviolet-pump-extremeultraviolet-probe experiments with isolated attosecond pulses will have to rely on tedious single-shot tagging approaches. It is fortunate that our results (see Fig. $4 \mathrm{~b}$ ) provide the required tag and thus serve this purpose too. Indeed, our method, besides its main task of intense isolated attosecond pulse generation, offers a versatile technique as a CEP diagnostic.

\section{Received 9 February 2007; accepted 7 September 2007; published 28 0ctober 2007.}

\section{References}

1. Hansch, T. W. A proposed sub-femtosecond pulse synthesizer using separate phase-locked laser oscillators. Opt. Commun. 80, 71-75 (1990).

2. Farkas, Gy. \& Toth, Cs. Proposal for attosecond light pulse generation using laser induced multiple-harmonic conversion processes in rare gases. Phys. Rev. A 168, 447-450 (1992).

3. Tzallas, P. et al. Direct observation of attosecond light bunching. Nature 426, 267-271 (2003).

4. Nabekawa, Y. et al. Interferometric autocorrelation of an attosecond pulse train in the single-cycle regime. Phys. Rev. Lett. 97, 153904 (2006).

5. Nabekawa, Y. et al. Conclusive evidence of an attosecond pulse train observed with the mode-resolved autocorrelation technique. Phys. Rev. Lett. 96, 083901 (2005).

6. Paul, P. M. et al. Observation of train of attosecond pulses from high harmonic generation. Science 292, 1689-1692 (2001).

7. Mairesse, Y. et al. Attosecond synchronization of high-harmonic soft X-rays. Science 302, $1540-1543$ (2003).

8. Hentschel, M. et al. Attosecond metrology. Nature 414, 509-513 (2002).

9. Kienberger, R. et al. Atomic transient recorder. Nature 427, 817-821 (2004).

10. Sansone, G. et al. Isolated single-cycle attosecond pulses. Science 314, 443-446 (2006).

11. Sola, I. J. et al. Controlling attosecond electron dynamics by phase-stabilized polarization gating. Nature Phys. 2, 319-322 (2006).

12. Baeva, T., Gordienko, S. \& Pukhov, A. Relativistic plasma control for single attosecond $\mathrm{x}$-ray burst generation. Phys. Rev. E 74, 065401(R) (2006).

13. Corkum, P. B. Plasma prospective on strong-field multiphoton ionization. Phys. Rev. Lett. 71, 1994-1997 (1993).

14. Lewenstein, M. et al. Theory of high harmonic generation by low-frequency laser fields. Phys. Rev. A 49, 2117-2132 (1994).

15. Kobayashi, Y. et al. 27-fs extreme ultraviolet pulse generation by high order harmonics. Opt. Lett. 23, 64-66 (1998)

16. Papadogiannis, N. A. et al. Two XUV-photon ionization of He through a superposition of higher harmonics. Phys. Rev. Lett. 90, 133902 (2003). 
17. Nikolopoulos, L. A. A. et al. Second order autocorrelation of an XUV attosecond pulse train. Phys. Rev. Lett. 94, 113905 (2005)

18. Sekikawa, T. et al. Nonlinear optics in the extreme ultraviolet. Nature 432, 605-608 (2004).

19. Nabekawa, Y. et al. Production of doubly charged helium ions by two-photon absorption of an intense sub-10-fs soft X-ray pulse at $42 \mathrm{eV}$ photon energy. Phys. Rev. Lett. 94, 043001 (2005).

20. Benis, E. P. et al. Frequency-resolved photoelectron spectra of two-photon ionization of He by an attosecond pulse train. New J. Phys. 8, 92 (2006).

21. Benis, E. P. et al. Two-photon double ionization of rare gases by a superposition of harmonics. Phys. Rev. A 74, 051402(R) (2006)

22. Miyamoto, N. et al. Observation of two-photon above-threshold ionization of rare gases by XUV harmonic photons. Phys. Rev. Lett. 93, 083903 (2004).

23. Corkum, P. B., Burnett, N. H. \& Ivanov, M. Y. Subfemtosecond pulses. Opt. Lett. 19, 1870-1872 (1994).

24. Baltuska, A. et al. Attosecond control of electronic processes by intense light fields. Nature 421, 611-615 (2003).
25. Tcherbakoff, O. et al. Time-gated high-order harmonic generation. Phys. Rev. A 68, 043804 (2003). 26. Tsakiris, G. D. et al. Route to intense single attosecond pulses. New J. Phys. 8, 19 (2006).

27. Antoine, P., L'Huillier, A. \& Lewenstein, M. Attosecond pulse trains using high-order harmonics. Phys. Rev. Lett. 77, 1234-1237 (1996).

28. Gaarde, M. B. \& Schafer, K. J. Space-time considerations in the phase locking of high harmonics. Phys. Rev. Lett. 89, 213901 (2002).

\section{Acknowledgements}

This work is supported in part by the European Community's Human Potential Program under contract MRTN-CT-2003-505138 (XTRA); MTKD-CT-2004-517145 (X-HOMES); the Ultraviolet Laser Facility (ULF) operating at FORTH-IESL (contract no. HPRI-CT-2001-00139) and the P14 COST programme.

Correspondence and requests for materials should be addressed to P.T.

Reprints and permission information is available online at http://npg.nature.com/reprintsandpermissions/ 